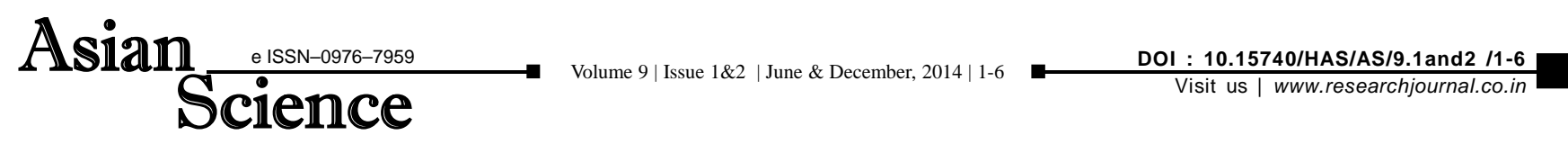

RESEARCH PAPER

\title{
A study on extent of utilization of maternal health care services through community health workers among rural women in Ruhango district at Rwanda
}

\author{
MUVANDIMWE EMMANUEL* AND J. VIJAYALAKSHMI \\ Department of Population Studies, Annamalai University, Annamalai Nagar, CHIDAMBARAM (T.N.) INDIA \\ (Email: vijmi1@gmail.com)
}

\begin{abstract}
The use of community health workers has been identified as one strategy to address the growing shortage of health workers, particularly in low-income countries. Community health workers (CHWs) are widely used to provide care for a broad range of health issues. The study is aimed to assess the extent of utilization of maternal health care services among rural women by CHWs. A sample of 120 respondents was randomly selected from 30 villages. Questionnaire method was used for data collection and analysis was done through frequency and percentage. All the respondents (100\%) were educated about maternal health by CHWs .Majority of respondents (85\%) was visited for 3 times by CHWs before delivery. Furthermore, 53 per cent of the respondents undergone check up for 4 times. Majority of respondents (98\%) were accompanied by CHWs to the health centre or hospital during delivery period. All those who had experienced complication (68\%) during delivery had taken treatment with the help of CHWs. All the respondents (100\%) were visited immediately at their homes after delivery by CHWs. In this paper, an attempt has been made to show how CHWs played a vital role in increasing the access to health care services among women and working within a community-based team to educate them on the importance of antenatal care and postnatal care with a skilled professional.
\end{abstract}

Key Words : Ante natal care, Delivery care, Community health workers, Health care services

View point paper : Emmanuel, Muvandimwe and Vijayalakshmi, J. (2014). A study on extent of utilization of maternal health care services through community health workers among rural women in Ruhango district at Rwanda. Asian Sci., 9(1\&2): 1-6.

\footnotetext{
* Author for correspondence

Muvandimwe Emmanuel, Department of Population Studies, Annamalai University, Annamalai Nagar, CHIDAMBARAM (T.N.) INDIA

(Email: muva2013@gmail.com)
} 